\title{
A GrC-Based Approach to Social Network Data Protection $^{\star}$
}

\author{
Da-Wei Wang, Churn-Jung Liau, and Tsan-sheng Hsu \\ Institute of Information Science, Academia Sinica, Taipei 115, Taiwan \\ \{wdw, liaucj, tshsu\}@iis.sinica.edu.tw
}

\begin{abstract}
Social network analysis is an important methodology in sociological research. Although social network data is very useful to researchers and policy makers, releasing it to the public may cause an invasion of privacy. In this paper, we generalize the techniques used to protect private information in tabulated data, and propose some safety criteria for assessing the risk of breaching confidentiality by releasing social network data. We assume a situation of data linking, where data is released to a particular user who has some knowledge about individual nodes of a social network. We adopt description logic as the underlying knowledge representation formalism and consider the safety criteria in both open-world and closed-world contexts.
\end{abstract}

\section{Introduction}

Social network analysis (SNA) is a methodology used extensively in social and behavioral sciences, as well as in political science, economics, organization theory, and industrial engineering $[6$. Though the analysis of social network data is valuable to researchers and policy makers, there may be a risk of privacy invasion. In SNA, researchers usually collect data by social surveys. Respondents to the surveys are typically anonymous; however, previous research on tabulated data has shown that simply ensuring the anonymity of respondents may not be sufficient to protect their privacy. The key point is that respondents could be reidentified by linking the anonymous data with some publicly available databases

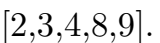

While each data record in a data table is completely defined by the attribute values of an individua 1 , a social network also contains relational data between individuals. In this sense, a social network is more general than a data table. In this paper, we provide a formal model of a situation where personal information not only contains attribute values, but also relational links to other individuals.

Several methods have been developed for tabulated data protection [8,9]. The main idea is to group the individuals with the same combination of public attribute values into a bin, or an information granule. Some qualitative and

\footnotetext{
* This work was partially supported by the Taiwan Information Security Center (TWISC) and NSC (Taiwan). NSC Grants: 94-2213-E-001-030 (D.W. Wang), 952221-E-001-029-MY3 (C.J. Liau), and 94-2213-E-001-014 (T-s. Hsu).

${ }^{1}$ An individual refers to a person whose privacy should be protected. 
quantitative safety criteria are then defined, according to the distribution of the confidential attribute values of individuals in the same information granule. In a social network, two individuals with the same public attribute values may still be distinguishable by their relationship with other individuals. Thus, to formulate information granules for social networks, we have to consider the attributes of individuals and the relationships between individuals. By generalizing the definition of information granules, we can extend the analysis for tabulated data to social network data.

The remainder of the paper is organized as follows. In the next section, we review a basic description logic. In Section 3, we discuss the representation of a social network with description logic languages. In such a representation, the social network data is transformed into an ABox, or an interpretation of the description logic based on the open-world or closed-world assumption. In Section 4, the transformations are described in detail and some safety criteria are defined. Finally, in Section 5, we present our conclusions.

\section{Description Logic - A Review}

Description logic (DL) was originally a subset of first-order logic specially designed for knowledge representation 2. In this section, we introduce a basic DL called $\mathcal{A L}$ (attributive language).

\subsection{Basic Syntax and Semantics}

The language $\mathcal{A L}$ was first introduced in 5 . The alphabets of $\mathcal{A L}$ consist of two disjoint sets, the elements of which are called concept names (atomic concepts) and role names (atomic roles) respectively. Following the notations in [1, we use the letters $A$ and $B$ for atomic concepts, the letter $R$ for atomic roles, and the letters $C$ and $D$ for concept terms. The concept terms of $\mathcal{A L}$ are formed according to the following syntactic rule:

$$
C::=A|\top| \perp|\neg A| C \sqcap D|\forall R: C| \exists R: \top .
$$

Note that, in $\mathcal{A L}$, negation can only be applied to atomic concepts, and only the top concept is allowed in the scope of an existential quantification.

The Tarskian semantics for $\mathcal{A L}$ assigns sets to concept names and binary relations to role names. Formally, an interpretation of $\mathcal{A} \mathcal{L}$ is a pair $I=\left(\Delta^{I}, \rrbracket \rrbracket_{I}\right)$, where $\Delta^{I}$ is a non-empty set called the domain of the interpretation and $\|\cdot\|_{I}$ is an interpretation function that assigns to each concept name a subset of $\Delta^{I}$ and to each role name a subset of $\Delta^{I} \times \Delta^{I}$. For brevity, we usually drop the subscript and superscript $I$. The domain of $\|\cdot\|$ can then be extended to all concept terms by induction as usual. In particular, we have

$$
\llbracket \forall R: C \rrbracket=\{x \in \Delta \mid \forall y((x, y) \in \llbracket R \rrbracket \Rightarrow y \in \llbracket C \rrbracket)\} .
$$

\footnotetext{
${ }^{2}$ Most of the notations and definitions in this section follow those introduced in [1].
} 


\subsection{Knowledge Representation by DL}

Within a knowledge base one can distinguish between intensional knowledge (i.e., general knowledge about the problem domain) and extensional knowledge, which is specific to a particular problem. A DL knowledge base is similarly comprised of two components - a "TBox" and an "ABox". The TBox consists of terminological axioms 3 that have the form

$$
C \sqsubseteq D(R \sqsubseteq S) \text { or } C \equiv D(R \equiv S),
$$

where $C, D$ are concepts (and $R, S$ are roles). An equality whose left-hand side is an atomic concept is called a definition, which is used to introduce a symbolic name for a complex description. An atomic concept not occurring on the lefthand side of an axiom is called primitive. A finite set of Definitions $\Sigma$ is called a terminology, or TBox, if no symbolic name is defined more than once; that is, if for every atomic concept $A$, there is at most one axiom in $\Sigma$ whose left-hand side is $A$. An interpretation $I=\langle\Delta, \rrbracket \cdot \rrbracket\rangle$ satisfies the axiom $C \equiv D($ resp. $C \sqsubseteq D)$ iff $\rrbracket C \rrbracket=\rrbracket D \rrbracket$ (resp. $\rrbracket C \rrbracket \subseteq \rrbracket D \rrbracket$ ).

An ABox contains the world description in the form of assertional axioms

$$
C(a) \text { or } R(a, b)
$$

where $C$ is a concept term, $R$ is a role name, and $a, b$ are constants for naming the individuals in the world. The domain of the interpretation function $\llbracket \cdot \rrbracket$ is extended to the constants occurring in an ABox such that $\llbracket a \rrbracket \in \Delta$ for any constant $a$. We assume that distinct individual names denote distinct objects (the unique name assumption). For the purpose of distinction, an extended interpretation is used to give a full assignment of meanings to constants, concepts, and roles. An extended interpretation $I=\langle\Delta, \rrbracket \cdot \rrbracket\rangle$ satisfies an assertion $C(a)$ (resp. $R(a, b)$ ) iff $\rrbracket a \rrbracket \in \rrbracket C \rrbracket$ (resp. $(\rrbracket a \rrbracket, \rrbracket b \rrbracket) \in \rrbracket R \rrbracket$ ). I satisfies the $\mathrm{ABox} \Phi$ if it satisfies each assertion in $\Phi$. It is also said that $I$ is a model of the assertion or the ABox. An assertion $\varphi$ is a logical consequence of an $\operatorname{ABox} \Phi$, written as $\Phi \models \varphi$, if for every interpretation $I, I \models \Phi$ implies $I \models \varphi$.

\section{DL-Based Representation of Social Network Data}

To represent social network data, we partition the atomic concepts (resp. roles) into two disjoint sets. The first set contains the easily-known (EK) atomic concepts (resp. roles), and the second consists of the sensitive atomic concepts (resp. roles). Let $\mathcal{L}$ be a DL languag $]^{\mathbb{A}}$. We use $\mathcal{L}^{e}$ to denote the sub-language of concept terms, which is composed of EK atomic concepts and roles only. The sub-language of other concept terms, i.e., $\mathcal{L}-\mathcal{L}^{e}$ is denoted by $\mathcal{L}^{s}$.

\footnotetext{
${ }^{3}$ For this reason, DL is also known as terminological logic or concept logic.

${ }^{4}$ Though we have only exemplified a DL language in the $\mathcal{A L}$ family, the results in this paper do not depend on the choice of any particular DL language.
} 
In this paper, we assume that there is a set of actual individuals $\left\{a_{1}, a_{2}, \cdots, a_{m}\right\}$ whose data is available in the data center and represented as an actual social network. For privacy reasons, the data center must mask off the identities of the actual individuals, so it is assumed that the actual individuals are replaced by nominal constants $u_{1}, u_{2}, \cdots, u_{m}$ in the released network data. The data center would like to release an anonymous network, obtained from a part or the whole of the actual social network by masking off the identities of the actual individuals.

Formally, an anonymous social network (based on a DL language $\mathcal{L}$ ) is defined as a labeled graph $G=(V, E, l)$, where $V$ is a set of nodes, $E \subseteq V \times V$ is a set of edges between the nodes, and $l$ assigns a subset of atomic concepts to each node and a subset of atomic roles to each edge. We can mark each vertex of $G$ distinctly with a nominal constant $u_{i}$. Without loss of generality, and by slightly abusing the notation, we can identify $V$ by $\left\{u_{1}, u_{2}, \cdots, u_{m}\right\}$.

The basic assumption is that a particular uset 5 may know (though perhaps only partially) some information about the actual individuals before the anonymous network is released, and wants to obtain the private data of these individuals by linking his a priori knowledge to the released social network data. The data administrator needs to assess the possibility that the user could link his $a$ priori knowledge to the released social network data to discover any individual's private information. Thus, it is important to model the user's a priori knowledge. However, the data administrator may not know the user's a priori knowledge. In such cases, the data administrator makes the worst-case assumption that all easily known information about the individuals is known to the user. Therefore, from the data administrator's perspective, the user's knowledge is represented as an ABox in which only concepts and roles from $\mathcal{L}^{e}$ and actual individuals appear. In addition, we assume that both the user and the data administrator use a common ontology, i.e., a TBox, to represent the background knowledge of the problem domain.

\section{Privacy Protection in Data Linking}

We now consider the DL-based representation of the released anonymous network, and formulate the notion of information granules based on that representation. There are two possible interpretations of the released anonymous network. One is based on the closed-world assumption (CWA) and the other on the openworld assumption (OWA). By CWA, we mean that the anonymous network $G=(V, E, l)$ is complete in the sense that if $C \notin l(u)$, then $u$ has the property $\neg C$; and if $R \notin l(u, v), u$ is not $R$-related to $v$. Thus, from the anonymous network $G=(V, E, l)$, we can obtain an interpretation $I_{G}$. In OWA, on the other hand, the anonymous network $G=(V, E, l)$ may be incomplete, so if $C \notin l(u)$, then $u$ may have the property $C$ or $\neg C$, and analogously for the roles. Thus, the social network can only be represented as an ABox, since DL typically adopts open-world semantics for the interpretation of an ABox.

\footnotetext{
${ }^{5}$ In this paper, "user" or "users" refers to anyone receiving social network data and having the potential to breach the privacy of individuals.
} 
In general, DL-based social network representations adopt the open-world assumption. However, in some special cases when only primitive concepts are considered, the CWA enables a more efficient representation of knowledge, since only positive information is needed.

In the following, we first present the safety criteria for CWA and then those for OWA. We also have to represent the user's knowledge available to the data administrator. In practice, the user's knowledge is rarely complete, so we always represent it by an ABox. Let $\Phi$ denote such an ABox throughout this section. The individual constants occurring in the ABox for the user's knowledge are the actual ones. It is assumed that the concepts and roles appearing in the ABox are all from $\mathcal{L}^{e}$, and the set of actual individuals occurring in the ABox is $\mathcal{A}$. Note that it is possible that the cardinality of $\mathcal{A}$ is less than the number of anonymous individuals in the social network, since the user may not have information about all actual individuals.

\subsection{Social Networks in a Closed World}

CWA has been adopted in much of the database literature. It is efficient for data representation, since only positive information is explicitly represented. However, due to an obvious technical reason, CWA is only applicable when the social network $G=(V, E, l)$ is restricted to a special form, where each element of $l(u)$ is primitive for all $u \in V^{6}$. In this subsection, we assume that the released social network meets this special restriction.

With CWA, an anonymous network can be transformed into an interpretation of DL. Formally, given a social network $G=(V, E, l)$ based on a DL language $\mathcal{L}$, we construct an interpretation $I_{G}=\left(V, \rrbracket \cdot \rrbracket_{I_{G}}\right)$ such that

1. the domain of the interpretation is the set of anonymous individuals $V$

2. $u \in \llbracket A \rrbracket_{I_{G}}$ iff $A \in l(u)$ for each primitive concept $A$

3. $(u, v) \in \llbracket R \rrbracket_{I_{G}}$ iff $R \in l((u, v))$ for each atomic role $R$

4. the interpretation of other non-primitive atomic concepts is determined by the axioms in the TBox of our problem domain.

Let $\Phi$ be an ABox representing the user's knowledge. Then, an extended interpretation $I=\left(V, \rrbracket \cdot \rrbracket_{I}\right)$ for $\Phi$ is said to be an extension of the interpretation $I_{G}$, written as $I_{G} \triangleright I$, if $\llbracket A \rrbracket_{I}=\rrbracket A \rrbracket_{I_{G}}$ for each concept name $A$ and $\llbracket R \rrbracket_{I}=\rrbracket R \rrbracket_{I_{G}}$ for each role name $R$. The set of $G$-based models for $\Phi$ is defined as

$$
\operatorname{Mod}(\Phi, G)=\left\{I \mid I_{G} \triangleright I \text { and } I \models \Phi\right\} .
$$

Note that a $G$-based model maps each actual individual appearing in $\Phi$ to a nominal constant in $G$. Such a mapping provides the user with a possible reidentification of these individuals in the anonymous network.

\footnotetext{
${ }^{6}$ If $B \equiv C \cup D$ is an axiom in the background TBox and $B \in l(u)$, but $C, D \notin l(u)$, then we obviously can not assume $\neg C \in l(u)$ and $\neg D \in l(u)$ simultaneously without contradiction.
} 


\subsection{Safety Criteria Under CWA}

Once the $G$-based models for the user's knowledge $\Phi$ are available, we can define different criteria to safeguard confidentiality when releasing an anonymous social network to a particular user.

The first safety criterion is the granule size (bin size) criterion, which was proposed in some pioneering works [4]. First, we define a granule for each actual individual $a \in \mathcal{A}$ as the set of possible anonymous individuals corresponding to $a$. Thus, the granule (or bin) for an actual individual $a \in \mathcal{A}$ with respect to $G$ and $\Phi$ is formally defined as

$$
[a]_{G}^{\Phi}=\left\{\rrbracket a \rrbracket_{I} \mid I \in \operatorname{Mod}(\Phi, G)\right\} .
$$

The pair $(\Phi, G)$ is said to satisfy the $k$-anonymity criterion if for each $a \in \mathcal{A}$, $\left|[a]_{G}^{\Phi}\right| \geq k$, where $|\cdot|$ denotes the cardinality of a set. The rationale behind the criterion is that the more individuals there are in a granule, the less likely it is that the user can re-identify to whom the anonymous individuals correspond.

It is shown in [3] that the granule size criterion may be not sufficient for privacy protection. Even though the user can not re-identify the anonymous individuals, he can sometimes deduce the sensitive information common to all individuals in a granule. To formulate a safety criterion for this situation, we assume $\mathcal{S C} \subseteq \mathcal{L}^{\mathcal{s}}$ to be a set of sensitive concepts that we would like to prevent users from knowing. Because the $G$-based models for $\Phi$ in fact determine all possible correspondence between the actual individuals and the anonymous individuals, an actual individual $a$ is known to have the property $C$ if $I \models C(a)$ for all $I \in \operatorname{Mod}(\Phi, G)$. Hence, $(\Phi, G)$ satisfies the epistemic safety criterion if for every $a \in \mathcal{A}$ and $C \in \mathcal{S C}$, there exists $I \in \operatorname{Mod}(\Phi, G)$ such that $I \not \forall C(a)$. It can be easily shown that $(\Phi, G)$ satisfies the epistemic safety criterion if $[a]_{G}^{\Phi} \nsubseteq \llbracket a \rrbracket_{I_{G}}$ for every $a \in \mathcal{A}$ and $C \in \mathcal{S C}$.

The two above-mentioned criteria are purely qualitative. Two quantitative criteria, called the average benefit and the total cost criteria, have been proposed for tabulated data [9]. These criteria can also be generalized to the current setting. In the average benefit model, we measure a privacy breach by estimating the average benefit the user can gain after receiving the anonymous social network. This is based on the assumption that the individuals' private information is valuable to the user; therefore, from the privacy protection viewpoint, the larger the average benefit, the less safe the social network data will be. On the other hand, the total cost model measures data safety by estimating the effort the user must expend to find the private information of every individual after receiving the anonymous social network. Thus, the higher the total cost, the harder it is for the user to find the individuals' private information.

The average benefit model is based on the information-theoretic measure of entropy [7. For each sensitive concept $C \in \mathcal{S C}$, the a priori probability of an arbitrary individual satisfying $C$ is denoted by $\operatorname{Pr}(C)$. It is sometimes assumed that value of $\operatorname{Pr}(C)$ can be obtained from some external knowledge sources, and is therefore available to both the user and the data center. If the assumption fails, the value of $\operatorname{Pr}(C)$ can be estimated by 


$$
\operatorname{Pr}(C)=\frac{|\{u \in V \mid C \in l(u)\}|}{m} .
$$

After receiving the released anonymous network data, the user knows the $a$ posteriori probability of an actual individual $a$ satisfying a sensitive concept $C$. This is defined by

$$
\operatorname{Pr}_{a}(C)=\frac{|\{I \in \operatorname{Mod}(\Phi, G) \mid I \models C(a)\}|}{\mid(\operatorname{Mod}(\Phi, G) \mid} .
$$

The user's information gain about $a$ regarding the sensitive concept $C$ is thus

$$
I G_{a}(C)=\max \left(\frac{\log \operatorname{Pr}(C)-\log \operatorname{Pr}_{a}(C)}{\log \operatorname{Pr}(C)}, 0\right) .
$$

Hence, the user's average information gain regarding $C$ is defined as

$$
I G(C)=\frac{\sum_{a \in \mathcal{A}} I G_{a}(C)}{|\mathcal{A}|} .
$$

Let us further assume bef $: \mathcal{S C} \rightarrow \Re^{+} \cup\{0\}$ is a function that maps each sensitive concept $C$ to the corresponding benefit the user can gain when he obtains some information about $C$. The value bef $(C)$ can also be seen as the degree of damage caused to an individual if it becomes known that he has the property $C$. Then, the average benefit to a user by receiving the social network data is $\mathcal{B}=\sum_{C \in \mathcal{S C}}$ be $f(C) \cdot I G(C)$. The larger the value of $\mathcal{B}$, the greater the amount of private information that is leaked.

Let us now turn to the total cost model, which estimates the cost to the user for completely knowing the private information of all individuals. It is assumed that the user can conduct an investigation of the actual individuals to determine whether any of them have the property $C$. If the user does not have any knowledge about these individuals, he has to investigate all of them. However, by utilizing the knowledge deduced from the released anonymous network, he could discover the private information of all individuals without investigating all of them. The total cost is based on the number of individuals he has to investigate in order to discover the private information of all individuals.

A constructive procedure is used to determine the total cost. Since each model in $\operatorname{Mod}(\Phi, G)$ stands for a possible correspondence between the actual and the anonymous individuals, the user can increase his knowledge by eliminating the impossible models via investigation.

Let us first fix a sensitive concept $C$. Then, for each actual individual $a \in \mathcal{A}$ and class of interpretations $\mathcal{M}$, define $\mathcal{M}_{a}^{+}=\{I \in \mathcal{M} \mid I \models C(a)\}$ and $\mathcal{M}_{a}^{-}=$ $\{I \in \mathcal{M} \mid I \not \models C(a)\}$.

Next, we enumerate all possible investigation sequences by using the investigation tree. Each node of the investigation tree denotes a string of actual individuals, which represents a sequence of individuals who have been investigated on that node. Thus, the root node is denoted by the empty string $\epsilon$. Let 
us define $\mathcal{M}_{\epsilon}=\operatorname{Mod}(\Phi, G), A_{\epsilon}=\mathcal{A}$, and $p_{\epsilon}=1$. Then, for each node denoted by $\lambda=\lambda^{\prime} \cdot a$ such that $a \in A_{\lambda^{\prime}}$, define

$$
\begin{gathered}
\mathcal{M}_{\lambda}=\left\{\begin{array}{l}
\left(\mathcal{M}_{\lambda^{\prime}}\right)_{a}^{+}, \text {if } C(a) \text { holds } \\
\left(\mathcal{M}_{\lambda^{\prime}}\right)_{a}^{-}, \text {if } C(a) \text { does not hold },
\end{array}\right. \\
A_{\lambda}=\left\{a \in A_{\lambda^{\prime}} \mid\left(\mathcal{M}_{\lambda}\right)_{a}^{+} \neq \emptyset \wedge\left(\mathcal{M}_{\lambda}\right)_{a}^{-} \neq \emptyset\right\},
\end{gathered}
$$

and

$$
p_{\lambda}=p_{\lambda^{\prime}} \cdot \frac{1}{\left|A_{\lambda^{\prime}}\right|} .
$$

Intuitively, $\mathcal{M}_{\lambda}$ and $A_{\lambda}$ respectively denote the set of remaining possible models and the set of individuals whose $C$-membership is not yet known; and $p_{\lambda}$ denotes the probability that the actual investigation sequence is $\lambda$. On each internal node $\lambda$, we assume that the next individual to be investigated is chosen uniformly from $A_{\lambda}$.

A node denoted by $\lambda$ is a leaf node if $A_{\lambda}=\emptyset$. Let $\mathcal{P}$ denote the set of all leaf nodes. Then, the expected number of investigations by the user is

$$
I V_{C}=\sum_{\lambda \in \mathcal{P}} p_{\lambda} \cdot \operatorname{len}(\lambda)
$$

where len $(\lambda)$ denotes the length of the string $\lambda$.

The rationale for taking the cost as a safety criterion is to prevent the user substantially reducing his investigation costs after receiving the released network data. To measure the extent of privacy leakage, we should consider the minimal effort the user must expend to find some private information; therefore, the expected total cost for the user to discover some private information after receiving the social network data should be $\mathcal{D}=\min _{C \in \mathcal{S C}} I V_{C}$. The larger the value of $\mathcal{D}$, the harder it is to breach privacy.

\subsection{Social Networks in an Open World}

An anonymous social network $G=(V, E, l)$ under OWA can be transformed into an $\operatorname{ABox} \Phi_{G}$ as follows:

$$
\begin{aligned}
\Phi_{G} & =\{A(u) \mid A \in l(u), u \in V\} \\
& \cup\{R(u, v) \mid R \in l((u, v)),(u, v) \in E\} .
\end{aligned}
$$

Consequently, we have an ABox, $\Phi$, for the user's knowledge and an ABox, $\Phi_{G}$, for the anonymous social network $G$. The user seeks a mapping from $\mathcal{A}$ to $V=\left\{u_{1}, \cdots, u_{m}\right\}$. Let $\iota: \mathcal{A} \rightarrow V$ be a 1-1 mapping; then $\iota\left(\Phi_{G}\right)$ is the resultant ABox obtained from $\Phi_{G}$ by replacing each anonymous individual $u$ occurring in $\Phi_{G}$ with $\iota^{-1}(u)$. The mapping $\iota$ is said to be a $(\Phi, G)$-possible matching if $\iota\left(\Phi_{G}\right) \cup \Phi$ is consistent with respect to the background TBox. Sometimes, we simply call it a possible matching. 


\subsection{Safety Criteria for OWA}

Once the notion of possible matchings is given, the definition of the granule size criterion is analogous to that under CWA. In fact, each $G$-based model corresponds exactly to a 1-1 mapping between $\mathcal{A}$ and $V$, which makes all assertions in $\Phi$ true. Under OWA, the granule (or bin) for an actual individual $a \in \mathcal{A}$ with respect to $G$ and $\Phi$ is formally defined as

$$
[a]_{G}^{\Phi}=\{\iota(a) \mid \iota \text { is a possible matching }\} .
$$

The pair $(\Phi, G)$ is said to satisfy the $k$-anonymity criterion if for each $a \in \mathcal{A}$, $\left|[a]_{G}^{\Phi}\right| \geq k$.

It is easy to define the epistemic safety criterion. Formally, $(\Phi, G)$ satisfies the epistemic safety criterion if for every $a \in \mathcal{A}$ and $C \in \mathcal{S C}$, there exists a possible matching $\iota$ such that $\iota\left(\Phi_{G}\right) \cup \Phi \not \models C(a)$.

For the average benefit model, the a priori probability $\operatorname{Pr}(C)$ may be still estimated by (3) or obtained from some external source. However, the a posteriori probability of an actual individual $a$ satisfying a sensitive concept $C$ is changed to

$$
\operatorname{Pr}_{a}(C)=\frac{\left|\left\{\iota \mid \iota\left(\Phi_{G}\right) \cup \Phi \models C(a)\right\}\right|}{\text { the number of possible matchings }} .
$$

The definitions of $I G_{a}(C)$ and $I G(C)$ are then exactly same as those given in (5) and (6) respectively.

We still use the investigation tree for the total cost model. For the root node, let $\mathcal{M}_{\epsilon}$ denote the set of all possible matchings, $\Phi_{\epsilon}$ be the empty set, and $A_{\epsilon}$ and $p_{\epsilon}$ be defined as above. Then, for each node denoted by $\lambda=\lambda^{\prime} \cdot a$ such that $a \in A_{\lambda^{\prime}}$, let

$$
\mathcal{M}_{\lambda}=\left\{\begin{array}{l}
\mathcal{M}_{\lambda^{\prime}}-\left(\mathcal{M}_{\lambda^{\prime}}\right)_{a}^{-}, \text {if } C(a) \text { holds } \\
\mathcal{M}_{\lambda^{\prime}}-\left(\mathcal{M}_{\lambda^{\prime}}\right)_{a}^{+}, \text {if } C(a) \text { does not hold }
\end{array}\right.
$$

where $\left(\mathcal{M}_{\lambda^{\prime}}\right)_{a}^{+}=\left\{\iota \in \mathcal{M}_{\lambda^{\prime}} \mid \iota\left(\Phi_{G}\right) \cup \Phi \cup \Phi_{\lambda^{\prime}} \models C(a)\right\}$ and $\left(\mathcal{M}_{\lambda^{\prime}}\right)_{a}^{-}=\left\{\iota \in \mathcal{M}_{\lambda^{\prime}} \mid\right.$ $\left.\iota\left(\Phi_{G}\right) \cup \Phi \cup \Phi_{\lambda^{\prime}} \models \neg C(a)\right\}$,

$$
\begin{gathered}
\Phi_{\lambda}=\left\{\begin{array}{l}
\Phi_{\lambda^{\prime}} \cup\{C(a)\}, \quad \text { if } C(a) \text { holds } \\
\Phi_{\lambda^{\prime}} \cup\{\neg C(a)\}, \text { if } C(a) \text { does not hold, }
\end{array}\right. \\
A_{\lambda}=A_{\lambda^{\prime}}-\left\{b \in A_{\lambda^{\prime}} \mid \mathcal{M}_{\lambda}=\left(\mathcal{M}_{\lambda}\right)_{b}^{+} \vee \mathcal{M}_{\lambda}=\left(\mathcal{M}_{\lambda}\right)_{b}^{-}\right\},
\end{gathered}
$$

and $p_{\lambda}$ be defined as above. The intuitive meanings of $\mathcal{M}_{\lambda}, A_{\lambda}$, and $p_{\lambda}$ are analogous to those of the CWA case, except that possible models are replaced by possible matchings. Note that $\mathcal{M}_{\lambda}=\left(\mathcal{M}_{\lambda}\right)_{b}^{+} \vee \mathcal{M}_{\lambda}=\left(\mathcal{M}_{\lambda}\right)_{b}^{-}$means that the $C$-membership of $b$ can be inferred from the known facts, so the investigation of $b$ is not necessary. In addition, $\Phi_{\lambda}$ contains the known facts discovered through the investigation of individuals in $\lambda$. The definitions of $I V_{C}$ and $\mathcal{D}$ then follow exactly the same form as above. 


\section{Conclusion}

In this paper, we generalize the privacy protection problem of microdata release in tabulated form to the social network form. A social network not only contains the attribute data of each individual, but also relational data between individuals. Therefore, the problem is more complicated than that encountered in tabulated data protection. We resolve it by employing a DL-based representation for social network data.

The safety criteria proposed in this paper enable a data center to assess the risk of breaching confidentiality by releasing social network data. However, some preventive action should be taken when the assessment shows that the direct release of the data is not safe. For tabulated data, some techniques such as generalization, suppression, and random perturbation have been studied extensively 4|319. In our future work, we will explore how to apply these techniques to social network data.

Another research problem is how to protect sensitive roles. In this paper, the safety criteria are mainly defined with respect to the protection of sensitive concepts. The protection of sensitive roles can be achieved indirectly. For example, if the data center wants to prevent the user from knowing $R(a, b)$, then it is sufficient to protect the sensitive concept $\exists R: \top$ for $a$. However, the criteria may be unnecessarily strict if it does not matter whether $a$ is $R$-related to anyone else apart from the particular $b$; therefore, the safety criteria can be further relaxed if defined with respect to the protection of roles directly.

\section{References}

1. F. Baader and W. Nutt. "Basic description logics". In F. Baader et al., editor, Description Logic Handbook, pages 47-100. Cambridge University Press, 2002.

2. Y.C. Chiang, T.-s. Hsu, S. Kuo, C.J. Liau, and D.W. Wang. Preserving confidentiality when sharing medical database with the Cellsecu system. International Journal of Medical Informatics, 71:17-23, 2003.

3. T.-s. Hsu, C.-J. Liau, and D.-W. Wang. A logical model for privacy protection. In Proc. of the 4th ISC, LNCS 2200, pages 110-124. Springer-Verlag, 2001.

4. P. Samarati. "Protecting respondents' identities in microdata release". IEEE Transactions on Knowledge and Data Engineering, 13(6):1010-1027, 2001.

5. M. Schmidt-Schauß and G. Smolka. "Attributive concept descriptions with complements". Artificial Intelligence, 48(1):1-26, 1991.

6. J. Scott. Social Network Analysis: A Handbook. SAGE Publications, 2 edition, 2000.

7. C.E. Shannon. "The mathematical theory of communication". The Bell System Technical Journal, 27(3\&4):379-423,623-656, 1948.

8. L. Sweeney. k-anonymity: a model for protecting privacy. International Journal on Uncertainty, Fuzziness and Knowledge-based Systems, 10(5):557-570, 2002.

9. D.W. Wang, C.J. Liau, and T.-s. Hsu. Medical privacy protection based on granular computing. Artificial Intelligence in Medicine, 32(2):137-149, 2004. 